\title{
Rotational period of GQ Lupi $i^{\star, \star \star}$
}

\author{
C. Broeg ${ }^{1,2,3}$, T. O. B. Schmidt ${ }^{1}$, E. Guenther ${ }^{2}$, A. Gaedke ${ }^{4,1}$, A. Bedalov ${ }^{1}$, R. Neuhäuser ${ }^{1}$, and F. M. Walter ${ }^{5}$ \\ ${ }^{1}$ Astrophysikalisches Institut und Universitäts-Sternwarte Jena, Schillergäßchen 2-3, 07745 Jena, Germany \\ e-mail: broeg@space.unibe.ch \\ 2 Thüringer Landessternwarte, Sternwarte 5, 07778 Tautenburg, Germany \\ 3 Max-Planck Institute for extraterrestrial Physics, Giessenbachstrasse, Garching, 85741, Germany \\ ${ }^{4}$ Hamburger Sternwarte, Gojenbergsweg 112, 21029 Hamburg, Germany \\ 5 Department of Physics and Astronomy, Stony Brook University, NY 11794-3800, USA
}

Received 22 November 2006 / Accepted 20 March 2007

\section{ABSTRACT}

\begin{abstract}
Aims. We wanted to determine the rotation parameters of GQ Lup A, thereby constraining the evolutionary history of the GQ Lup system.

Methods. We have undertaken a photometric monitoring campaign on GQ Lup A consisting of two epochs spaced one year apart. We also searched the photometric archives to enlarge the data set.

Results. We were able to determine the photometric period $(8.45 \pm 0.2$ days $)$ in both epochs in several photometric bands. This periodicity could also be found in some of the archival data. The combined false-alarm probability is 0.015 . The variation is most likely caused by hot spots on the surface of GQ Lup A. This, combined with high-resolution spectra $(v \sin i)$ allows calculation of GQ Lup A's inclination $\left(i=27 \pm 5^{\circ}\right)$. Radial velocity data also contains this period but is inconclusive. Nevertheless, the RV data supports the interpretation that hot spots cause the photometric variation. We use the known $K$-band variability, amplitude, and phase of GQ Lup A together with a new image of GQ Lup A+b, taken quasi-simultaneously with our monitoring of the star, to confirm the magnitude and, hence, luminosity of the companion.
\end{abstract}

Key words. stars: rotation - stars: fundamental parameters - stars: starspots

\section{Introduction}

Neuhäuser et al. (2005) recently discovered a sub-stellar companion around the classical T Tauri star GQ Lup A in the Lupus star forming region by direct imaging. The companion has a separation of $0.7^{\prime \prime}$ or $\sim 100 \mathrm{AU}$ (see also Mugrauer \& Neuhäuser 2005). This companion may have formed closer in, and been moved to a larger separation by interaction with a more massive inner companion (Debes \& Sigurdsson 2006; Boss 2006). Therefore the question arises whether GQ Lup harbors additional companions at closer distances which could be found by radial velocity techniques. Unfortunately, classical T Tauri stars are very active and can exhibit strong spot activity (Hoffmeister 1965). This can mimic radial velocity (RV) signals of an orbiting planet. This has been studied in detail by Saar \& Donahue (1997) and Saar et al. (1998). Neuhäuser et al. (1998) monitored the weak-line T Tauri star Par 1724 in Orion and attributed its RV variation to a cool spot. Paulson et al. $(2002,2004)$ used their precise RV-data of the Hyades stars to investigate the cause of the RV-scatter. They concluded that the scatter is mainly caused by spots, and that plage regions are less important. This result

* Based on observations obtained on Cerro Paranal, Chile, in ESO program 075.C-0710(C) and on La Silla, Chile, in ESO programs 074.C-0034(A),075.C-0710(E), 075.C-0710(F), 075.C-0202(A), 076.C-0010(A) as well as with ANDICAM of the SMARTS consortium.

$\star \star$ Photometry data of epochs I and II are only available in electronic form at the CDS via anonymous ftp to

cdsarc.u-strasbg.fr $(130.79 .128 .5)$ or via

http://cdsweb.u-strasbg.fr/cgi-bin/qcat?J/A+A/468/1039 is confirmed by RV-monitoring of the very active star EK Dra (König et al. 2005). The principle effect of dark spots on spectroscopy is that the reduced continuum level at the velocity of the spot leads to an apparent emission peak at that velocity, crossing the line profile as the spot transits the photosphere.

As part of an investigation of GQ Lup we undertook a photometric monitoring campaign to search for a photometric period of the primary. If this period can be ascribed to the rotation of the star, this allows - in combination with the $v \sin i$ from spectra - the determination of the inclination of GQ Lup A. This inclination is very likely also the inclination of the disk around GQ Lup A, and possibly also the inclination of its companion's orbit.

\section{Observations}

\subsection{Photometric monitoring}

We carried out a photometric monitoring campaign at the $1.3 \mathrm{~m}$ telescope of the Cerro Tololo Inter-American Observatory (CTIO), Chile, using the ANDICAM dual-channel photometer of the SMARTS consortium. The ANDICAM obtains simultaneous optical and near-IR imaging by splitting the beam with a dichroic filter and directing the light to two independent cameras. Observations were taken at two epochs. Over the 25 days from April 6 to May 1, 2005 (epoch I), data were taken on 15 nights (13 in the optical). We observed through the $R, I, J$, and $K$ filters. We obtained 3 consecutive images in each filter each night. The $J$ and $K$ images are dithered. In all, there are $39 R$ and $I$ and $45 J$ and $K$ band images. These observations 


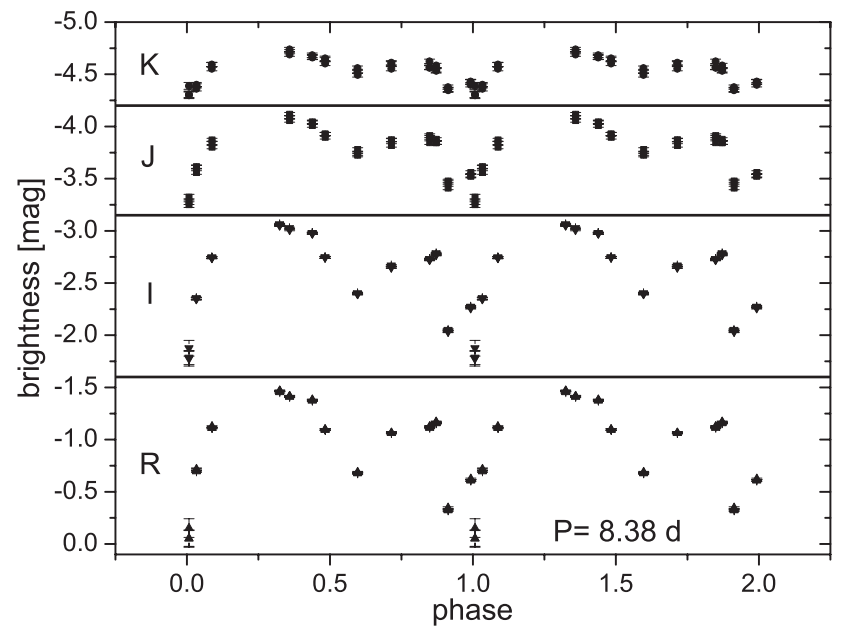

Fig. 1. Light curve of GQ Lup in the $R, I, J$, and $K$ broad band filters phased to a period of 8.38 days. The same pattern can be observed in all 4 filters and the amplitude decreases from $R$ to $K$ band. The most likely cause for this behavior is activity on the rotating stellar surface such as hot spots. The data are from April 2005 (epoch I).

were repeated one year later (epoch II) using the $V, R, J$, and $K$ filters. In the 17 days from May 9, 2006 to May 26, 2006, 25 sets of images were taken on 15 nights. Each IR exposure contains 3 dithered images, so there are 75 IR exposures per filter. Some of these images were later rejected by the differential photometry algorithm.

The optical ANDICAM data are processed (over-scan subtraction and flat-fielding) prior to distribution. Aside from a $2 \times 2$ re-binning, the IR data are delivered raw. The flat field images are generated from the dome flats obtained about every other night. We generated a sky image by taking the median of the dithered images. The sky image is then subtracted from the individual frames. We combine the individual $J$ and $K$ images using a shift and add technique. We performed aperture photometry to obtain the instrumental magnitudes. The resulting magnitudes were input into the differential photometry algorithm by Broeg et al. (2005) to obtain differential magnitudes for GQ Lup A.

We have made the measurements for both epoch I and epoch II available on-line at CDS. The archive contains the following information for each observed filter: the Julian date, the differential magnitude, and the calculated error as described in Broeg et al. (2005).

We used the differential magnitudes, to search for periods in all filters separately using the string-length method (SLA) (see Burke et al. 1970; Dworetsky 1983) as well as the discrete Fourier transform method (DFT).

Applying the string-length method to epoch I, all four filters produced a minimal string-length at the same period of 8.3 days. Using the Fourier transform, on the other hand, all filters gave the peak at a frequency of $0.117915 \mathrm{~d}^{-1}$ corresponding to a period of 8.48 days. Except for the alias frequency of $0.88 \mathrm{~d}^{-1}$, no other powers appear in the Fourier transform, and no other significant minima in the string-length algorithm.

The resulting period of our first epoch is therefore the mean value, $P=8.38 \pm 0.2$ days.

The light-curve phased to the best SLA period of 8.38 days is shown in Fig. 1. It is apparent that the photometric variability is of similar shape in all filters. The decrease in amplitude with increasing wavelength $[R(1.41 \mathrm{mag}), I(1.29 \mathrm{mag}), J(0.87 \mathrm{mag})$, $K(0.44 \mathrm{mag})]$ suggests that the variability is caused by hot or
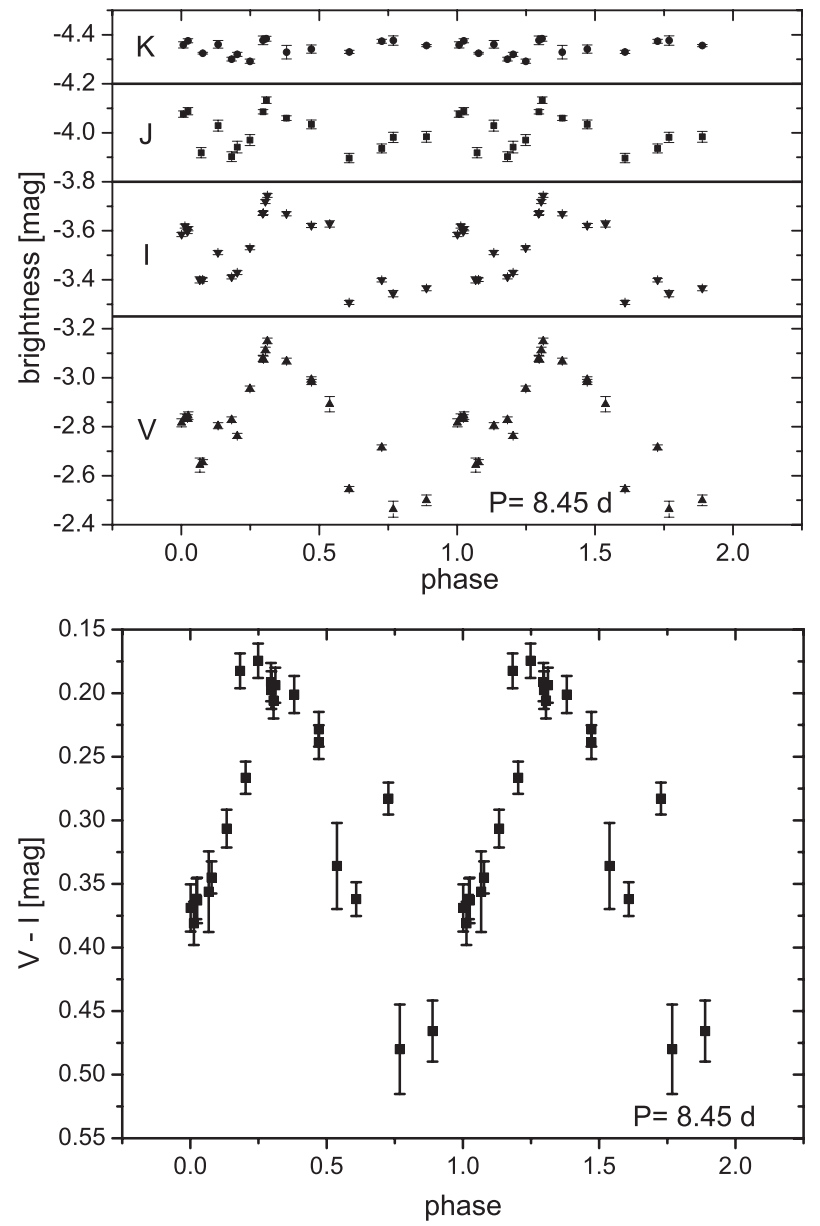

Fig. 2. Upper panel: light curve of GQ Lup in the $V, I, J$, and $K$ broad band filters phased to a period of 8.45 days. The same pattern can be observed in all 4 filters and the amplitude decreases from $V$ to $K$ band. Lower panel: the photometric color $V-I$ of GQ Lup A. A large value suggests a lower black-body temperature. The temperature correlates well with the brightness variation. This is strong evidence that temperature variations (e.g. hot/cold spots) are causing the brightness variation. The amplitude and shape of the light curve have changed in the year since the observations in Fig. 1. These data are from May 2006 (epoch II).

cold spots on the surface of the rotating star. Thermal emission from areas of different temperature (i.e. hot spots in the colder photosphere) will exhibit a greater flux difference at shorter wavelengths than at long wavelengths.

We repeated the above period search for epoch II. The shape and amplitude of the light curve have changed. The string-length algorithm in the $V$-band gives only two significant minima at 8.6 and 8.45 days. The $I$-band only has one minimum at 8.45 days. Because of the decrease in amplitude from epoch I to II, the amplitude in the infrared data is too small to permit a period determination. Nevertheless, the second epoch confirms our period from epoch I. Figure 2 shows the second epoch observations phased to the 8.45 days period. Additionally, the lower panel shows the $V-I$ color. Again, the correlation of color and brightness change indicates that temperature changes are the cause for the brightness variation. The most likely cause is hot spots on the surface of the star. This is indicated by the large amplitude of variation. Cold spots do not usually produce such large amplitudes (compare e.g. Herbst 1989, or Neuhäuser et al. 1998). 
Table 1. Literature data with photometry on GQ Lup. We have combined data that was taken close together.

\begin{tabular}{cllcl}
\hline \hline group & source, designation & year & \# measurements & comments \\
\hline 1 & Schwartz \& Noah (1978), SN & March 1977 & 4 & \\
1 & Appenzeller et al. (1978), A & July 1977 & 8 & \\
1 & Bertout et al. (1982), B & April 1978 & 5 & $\begin{array}{l}\text { conversion from 13 color system } \\
\text { (Johnson \& Mitchell 1975), Fe II features }\end{array}$ \\
& & & 13 & \\
2 & Covino et al. (1992), C & May 1990 & 123 & AAVSO \\
3 & Waagen (2005) & July 2001-June 2005 & 2 & only 2 points \\
- & Mundt \& Bastian (1980) & January 1979 & 34 & large scatter, no period \\
- & Kardopolov \& Filipev (1985) & June-July 1985 & no period found \\
- & Herbst (1985) & 1985 & 22 & \\
\hline
\end{tabular}

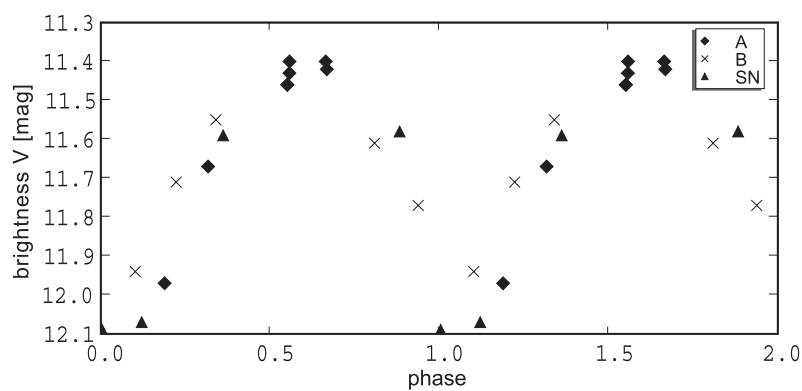

Fig. 3. Light curve of GQ Lup in the $V$ band taken from literature data in group 1 (1977/78). The data have been folded assuming a period of 8.41 days. In all 3 data sets, the same single peak structure can be observed.

Combining both epochs, we conclude that GQ Lup A has a photometric period of $P=8.45 \pm 0.2$ days.

Also, photometric data were taken in ESO run 075.C-0710 in May 2005, but data are available only from one night, so that they are not useful for constraining the period.

\subsection{Photometry from archive data}

We also looked for photometry on GQ Lup in archive data. It has been observed by a number of groups. We also obtained data from the AAVSO (Waagen 2005) project. All sources are listed in Table 1.

Since there were only a few observations in some of the publications, we combined those measures from the same observing seasons. Combining all of the data is not recommended since activity driven variability might change over time as is confirmed by our own photometric study. We used only data in the $V$ band because most of the data are in that band.

Again, we searched for a periodic signal with both the SLA and the DFT. In the combined data of group 1, the DFT gives the strongest signal at a period of $8.41 \mathrm{~d}$. The resulting light curve shows a single peak, see Fig. 3. In the SLA, however, this period is only one among several probable candidates. This period could also be found in group 2 (from 1990) but it was not the strongest signal. The phased light curve shows a double peak and the amplitude of variation is much larger (see Fig. 4) which compares well with our new data (see Fig. 1). One possible explanation is that a second strong spot has appeared on the surface of the star during the decade between the observations. It is equally likely that a more complicated spot pattern has evolved over that time period. The timescale is rather short for spot-caused brightness variation. Nevertheless, a combination of more than one spot can lead to the observed light curve.

Additionally, we analyzed data from the American association of variable star observers (AAVSO), group 3. They provided

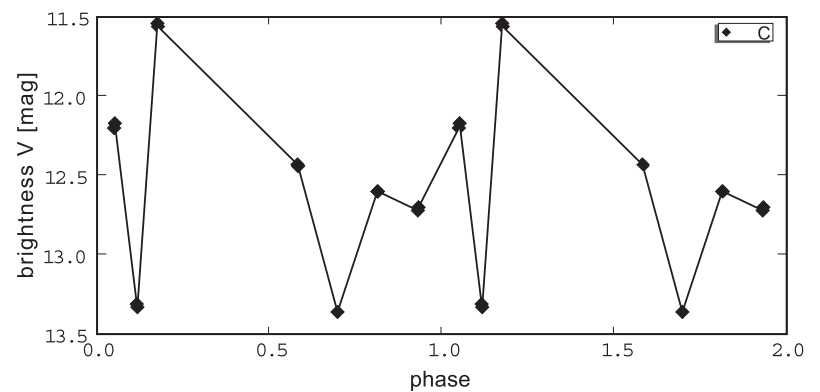

Fig. 4. Light curve of GQ Lup in the $V$ band taken from literature in group 2 (1990). The data have been folded assuming a period of 8.41 days. The light-curve now appears double-peaked as in April 2005. A line was added to guide the eye.

us with a total of 123 measurements. Unfortunately, those measurements are spread out over a period of 4 years. This leads to a Nyquist frequency of 0.07 suggesting that periods smaller than 14 days cannot be extracted from the data. Even when looking at subsets of the data that lead to a Nyquist period of smaller than 8 days, the 8.4 day period could not be confirmed in those data. There are many candidates in both the DFT and the SLA method, and the 8.4 day period is present in most subsets, but it is impossible to tell which period is correct or if any period is significant at all.

The other observations in the archives did not exhibit any convincing periodic signal.

In sum, all archival data taken together strongly hint at a period in the vicinity of 8.4 days. Furthermore, there is strong evidence that the active photosphere of GQ Lup has changed in the 1980's in-between the observations. This change in activity might be the reason why we could not determine any period in the AAVSO data which cover a time-span of 4 years. Taken together, the archival data confirm our photometric period of GQ Lup A from our photometric monitoring campaign.

\subsection{Lomb-Scargle periodogram analysis}

To quantify the periodicity of the data, we performed a LombScargle periodogram analysis (Scargle 1982; Horne \& Baliunas 1986). To determine the false-alarm probability (hf. FAP) we averaged measurements taken each night, so that there is at most one measurement per night as suggested in Horne \& Baliunas (1986).

As expected, no single observing run provides significant power in the Lomb-Scargle periodogram. Therefore we combined our two epochs to determine the significance of the period. We proceeded as follows: A) First, the Lomb-Scargle periodogram for each epoch is calculated in the same frequency range. B) Next, the power is converted to a FAP using the 


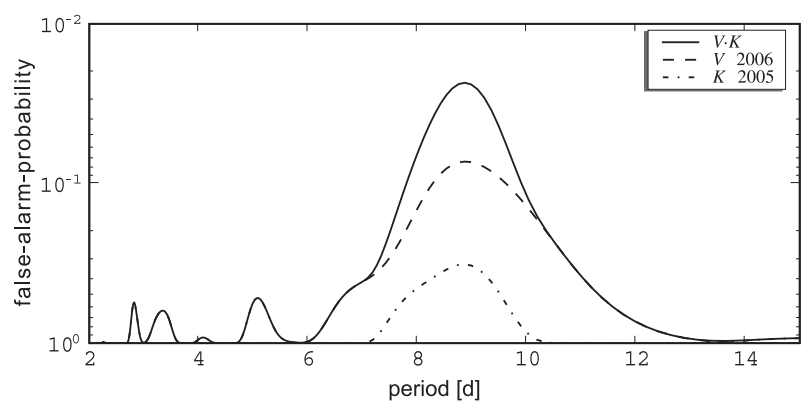

Fig. 5. False-alarm probabilities according to Scargle (1982) for the 2005 and 2006 observations (dashed lines). We consider both observations independent measurements and can therefore multiply the FAPs of both runs (solid line). The combined FAP is $2.3 \%$ at $P=8.86$ days. When combined with the literature data from group 2 the FAP is lowered to $1.5 \%$.

number of independent frequencies according to Horne \& Baliunas (1986). C) In a final step the FAPs at each frequency are multiplied to obtain the true FAP of both epochs taken together.

The combined FAP of the $2006 \mathrm{~V}$ and $2005 \mathrm{~K}$ observations, shown in Fig. 5, is $2.3 \%$ (minimum at $8.87 \mathrm{~d}$ ). Admittedly, this value is not very small. If we further add the 1992 literature data, group 2 (from Table 1), the FAP is lowered to $1.5 \%$ (minimum at $8.99 \mathrm{~d}$ ). Unfortunately, we cannot combine the data with group 1 because in group 1 there are not enough measurements in each subgroup to determine the Horne number of independent frequencies which is necessary to obtain a meaningful conversion from Lomb-Scargle power to FAP. Nevertheless, the group 1 data support the 8.5 day periodicity even though we cannot quantify the contribution. A combination of our $V$ and $K$ data with the HARPS radial velocity measurements (see Sect. 2.4) leads to a FAP of 0.004 (minimum at $8.64 \mathrm{~d}$ ).

The Lomb-Scargle analysis therefore confirms a periodic signal with a periodicity in the range from 8 to 10 days with a FAP of $1.5 \%$. However, it is ill-suited to determine the exact value of the period when confronted with non-sinusoidal input as a frequency shift must be expected in that case. In the extreme case of a double peaked structure (e.g. two peaks of different height and width), the Lomb-Scargle periodogram will even give a higher power at twice the true frequency and the location of the peak at the true frequency will be shifted. Therefore we use the SLA to determine the value of the frequency and keep the value of the photometric period as determined in the last section.

\subsection{Radial velocity measurements}

We observed GQ Lup A with the ESO echelle spectrograph FEROS $^{1}$ on the $1.5 \mathrm{~m}$, and later on the $2.2 \mathrm{~m}$-telescope at La Silla. The spectra cover the wavelength region between about $3600 \AA$ and $9200 \AA$, and have a resolution of $\lambda / \Delta \lambda=48000$. The standard MIDAS pipeline for FEROS was used for bias subtraction, flat-fielding, scattered light removal, echelle order definition and extraction, and wavelength calibration of the spectra. Precise measurements of the radial-velocity (RV) have then been carried out by cross-correlating the object spectra with spectra of the RV standard HR 5777 observed with the same instrument, using the IRAF ${ }^{2}$ RV package. The RV of HR 5777 is known to

\footnotetext{
${ }^{1}$ Fiber-fed Extended Range Optical Spectrograph.

${ }^{2}$ Image Reduction and Analysis Facility from the National Optical Astronomy Observatories (NOAO).
}

Table 2. Summary of radial velocity data.

\begin{tabular}{lll}
\hline \hline Instrument & Time & \# meas. \\
\hline HARPS (this paper) & Feb.-March 2005 & 7 \\
HARPS (this paper) & May 5, 2005 & 8 \\
FEROS (this paper) & March 1999-March 2000 & 3 \\
FEROS (Melo 2003) & July 1998-May 2000 & 6 \\
\hline
\end{tabular}

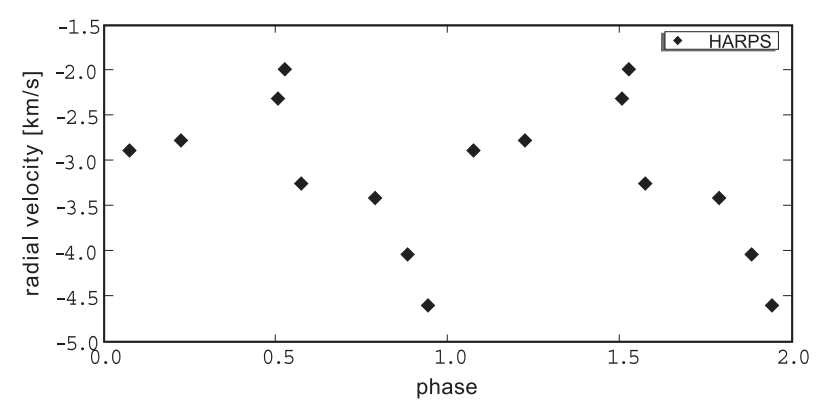

Fig. 6. Radial velocity curve showing the averaged HARPS data phase folded with an assumed period of 8.4 days. Despite the small time difference to the CTIO 2005 data (see Fig. 1) of only 50 days the phase cannot be compared to the photometry light curves. The error in the period determination of 0.2 days leads to a offset in phase of 0.2 making a comparison meaningless.

be stable, and has been determined with an accuracy of $55 \mathrm{~ms}^{-1}$ (Murdoch et al. 1993).

In addition, we have obtained HARPS $^{3}$ data with the ESO $3.6 \mathrm{~m}$ telescope on La Silla, Chile. The ESO pipeline was used to reduce the HARPS data, and to determine the RVs.

Furthermore we took the published RV data on GQ Lup from Melo (2003). All RV-data used are summarized in Table 2.

We proceeded as follows in searching for a periodic signal in the RV-data: Due to the 5-year time-span and the fact that we used two different instruments, it was questionable whether all data could be used in one data set. Therefore we started searching for periods in the HARPS data only. The SLA gives the smallest value for the period of 8.596 days. Combining the data with the FEROS data, there is an equally strong signal at around 4.92 and 8.4924 days. When looking at the FEROS data only, no period stands out. We cannot exclude that there is a slight offset in the zero point $\left(\$ 1 \mathrm{~km} \mathrm{~s}^{-1}\right)$ in the RV data taken from literature in Table 2. For this reason, and because of the change in spot pattern suggested by the photometric data, it is probably not feasible to combine the HARPS and FEROS data, so we'll ignore the periodicity in the combined data.

In sum, the RV data alone does not constrain the period. It appears clear that a periodic signal exists in the data but multiple frequencies are present. Taken together with the photometric data, it is likely that the 8.45 day period in the photometric data and the possible $\sim 8.6$ day period in the RV data are caused by the same mechanism, namely the rotation of large star spots on the surface of the star. This is supported by the amplitude of the RV signal $\left(\sim 3 \mathrm{~km} \mathrm{~s}^{-1}\right)$. Guenther et al. (2006) find that CTTS and WTTS show RV-variations of $\leq 3 \mathrm{~km} \mathrm{~s}^{-1}$ due to stellar activity. This value is in good agreement with the results from Saar et al. (1998), if one takes the larger $v \sin i$, and the larger spot-coverage of the young stars into account.

The HARPS RV measures phase-folded on the hypothetical period of 8.4 days is shown in Fig. 6 .

\footnotetext{
${ }^{3}$ High Accuracy Radial velocity Planet Searcher.
} 
While we cannot use the RV data as an independent confirmation of our photometric period, we can gain insight on possible stellar companions of GQ Lup A: we can certainly exclude an object causing RV-variations with a (semi-amplitude) larger than $2 \mathrm{~km} \mathrm{~s}^{-1}$. In total, the object was observed for 7 years, if we also take the published observations into account. Assuming a typical eccentricity of a binary of 0.3 , we can thus exclude a companion that is more massive than $0.1 M_{\odot}$ with a semi-major axis of $\leq 2.6 \mathrm{AU}$.

\subsection{Inclination of GQ Lup A}

If caused by star spots, the photometric period should be equal to the rotational period of GQ Lup A (neglecting differential rotation). This assumes that we see the effects of hot (or cold) spots on the surface of GQ Lup A. If we see hot areas in the disk on the other hand, e.g. at the inner edge of the disk, the rotational period might be different from the photometric period.

Because of the large amplitude in the photometric signal and because the photometric signal is also present in the optical bands, we will for now assume the variations are due to spots. Since we know the rotational period we can calculate GQ Lup A's inclination if we know its $v \sin i$. Guenther et al. (2005) give the $v \sin i=(6.8 \pm 0.4) \mathrm{km} \mathrm{s}^{-1}$. We know the luminosity $L=1.58 \pm 0.18 L_{\odot}$ and the effective temperature $T_{\text {eff }}=4060 \mathrm{~K}$ (both values from Neuhäuser et al. 2005, $T_{\text {eff }}$ from spectral type using Kenyon \& Hartmann 1995). Given the spectral type of the star, its uncertainty ( \pm one sub-class) and systematic uncertainties in the spectral type temperature conversion, we estimate the error of the effective temperature in GQ Lup A to be $\pm 300 \mathrm{~K}$.

This allows calculation of GQ Lup A's radius using the Stefan-Boltzmann law. Using the above values we get a radius of $(2.55 \pm 0.41) R_{\odot}$. Now we can calculate the equatorial surface velocity:

$v=2.55 R_{\odot} \frac{1}{8.45 \mathrm{~d}} 2 \pi \simeq(15.2 \pm 2.4) \mathrm{km} \mathrm{s}^{-1}$.

For the error estimation we use the conservative errors as given in the text above (for $L$ and $T_{\text {eff }}$ ) and an error in the rotational period of 0.2 days. This gives a $\sin i$ of 0.45 and an inclination $i \sim(26.6 \pm 4.8)^{\circ}$.

In sum, we derive GQ Lup A's inclination to be $i \sim(27 \pm$ $5)^{\circ}$. This result is obtained under the assumption that the $v \sin i$ measurement and the photometric variability are caused by the same phenomenon, e.g. spots on the surface of the star. If that is the case, we have an almost pole-on view onto the star; this is probably also the inclination of the disk around GQ Lup A, and possibly also of the orbit of GQ Lup b. In this case, using the projected separation of GQ Lup $b$ as $\sim 100 \mathrm{AU}$ from Neuhäuser et al. (2005), the true orbital separation would be $\sim 112$ AU.

Because of strong infrared excess emission, it appears very probably that GQ Lup A is surrounded by a strong and massive circumstellar disk. See e.g. Guenther (2006) for the spectral energy distribution of GQ Lup A. Batalha et al. (2001) argued that the disk is seen near edge-on because of variable extinction towards GQ Lup A. However, GQ Lup A shows not only hard, but also soft X-ray emission (see Krautter et al. 1997); this is atypical for T Tauri stars with disks, see Neuhäuser et al. (1995). The latter authors argued that the disks are seen near pole-on in such cases, so that soft X-rays are not completely extincted. This interpretation may apply for GQ Lup A. This is supported by new models (see Jardine et al. 2006; Gregory et al. 2006) that predict accretion near the equator caused by a different magnetic configuration in classical T Tauri stars as compared to main sequence stars. They also predict small filling factors which is in agreement with the shape of our light curves. Spots near the poles would most likely not produce a large variation when the star is seen pole-on.

\section{Relevance for GQ Lup b}

Neuhäuser et al. (2005) determined the magnitude of GQ Lup b by relative photometry compared to GQ Lup A. They found a brightness difference of $6.00 \pm 0.15 \mathrm{mag}$ in $K$. They did not take into account the possible variability of star A, but used its 2MASS value $(K=7.096 \pm 0.020 \mathrm{mag})$. The variability of star A would introduce an additional uncertainty (or rather a small shift when knowing the phase and correcting for this effect) not taken into account in Neuhäuser et al. (2005).

We obtained a new image of GQ Lup A and b on May 27, 2005 between 03:30h and 04:17h UT, again using NACO at the VLT/Yepun, as in Neuhäuser et al. (2005). We have reduced the data as in 2004.

Using the light curve from the $K$-band photometric monitoring of GQ Lup A during April 2005, we can predict the $K$-band magnitude of GQ Lup A during the NACO observation on 27 May 2005. However, due to the error in the period (0.2 days) and due to the fact, that the NACO observation falls into the narrow minimum seen in Fig. 1 (so that it could easily be brighter), a precise prediction is not possible. We predict for GQ Lup A a $K$-band magnitude of $K=7.00$ to $7.25 \mathrm{mag}$ for May 27, 2005, 04h UT.

We corrected from differential to apparent magnitude by using three stars from 2MASS in the GQ Lup field observed on a photometric night during our April 2005 monitoring. The offsets between the differential magnitudes and the 2MASS magnitudes of these three stars agree to $<1 \%(0.01 \mathrm{mag})$, so that they can be assumed to be long-term constant.

The magnitude difference between GQ Lup A and b on the image from May 27, 2005 is $6.10 \pm 0.15$ mag. With $K=7.00$ to 7.25 mag for star A, we find the magnitude of GQ Lup b to be $K=13.2 \pm 0.2 \mathrm{mag}$ (on May 27, 2005). Using $B C_{\mathrm{K}}=$ $3.0 \pm 0.1 \mathrm{mag}$ based on a revised temperature for GQ Lup b of $2600 \pm 100 \mathrm{~K}$ obtained from higher resolution $J_{-}, H$-, and $K$-band VLT/Sinfoni spectra (Seifahrt et al. 2007), and a distance of $140 \pm 50 \mathrm{pc}$, its luminosity will be $\log \left(L / L_{\odot}\right)=-2.29 \pm$ 0.41 . This is consistent with the values given in Neuhäuser et al. (2005) and Seifahrt et al. (2007).

We note that the same problem of possible variability of the primary can have occurred in the determination of the magnitude and, hence, luminosity and mass, of companions of other young stars such as, most recently, 2M1207 (Chauvin et al. 2005a), AB Pic (Chauvin et al. 2005b), and AB Dor (Close et al. 2005). The latter is known to be one of the most variable stars on the sky.

\section{Summary}

With our photometric monitoring, supplemented by archival data, we have determined the photometric period of GQ Lup A with a FAP of 0.015 . Since the amplitude of the brightness variation is strongly dependent on the wavelength of the filter, we conclude that the brightness variations are caused by hot or cold spots on the surface of the star. This implies that the photometric period is equal to the rotational period of GQ Lup A. Because of the large amplitude of the variations (1.5 mag in $R$ in 2005) 
hot spots are likely to be the cause. Hot spots may be a consequence of on-going accretion from the disk onto the star. We witnessed large changes in the activity pattern over a one year timescale. The amplitude of variation dropped by a factor of two in-between the two epochs.

The archival data provides additional evidence that the activity pattern of the star is constantly changing. It has changed qualitatively from 1979 to 1990 and finally to 2005/2006.

The radial velocity data also show one possible period near the photometric period, but it is not possible to exclude other frequencies. Nevertheless, the RV amplitude is in agreement with hot spots being the cause for the photometric variation.

Combining all the measurements, we conclude that $8.45 \pm$ 0.2 days is the photometric period and most likely also the rotational period of GQ Lup A. It is the only period that occurs in all data sets. A rotation period of 8.4 days is quite typical for $\mathrm{T}$ Tauri stars with disks. Because of the large time-spread of the different data sets, there is no phase coherence between different data sets and we cannot determine the period more precisely by combining the data sets.

We use the known periodicity together with new photometry data on GQ Lup A and b to refine the luminosity of GQ Lup b. It is confirmed at $\log L / L_{\odot}=-2.41 \pm 0.41$ (instead of $-2.37 \pm 0.41$ previously). The conclusions in Neuhäuser et al. (2005) remain valid.

Assuming that the brightness variations are caused by spots on the surface of GQ Lup A, we derive GQ Lup A's inclination to be $i=(27 \pm 5)^{\circ}$. This is likely also the inclination of its disk and companion's orbit. The semi-major axis of the orbit of GQ Lup b is $\sim 112 \mathrm{AU}$.

Acknowledgements. We thank the referee for his suggestion to include the Lomb-Scargle periodogram analysis which allows us to provide quantitative FAPs.

We acknowledge with thanks the variable star observations from the AAVSO International Database contributed by observers worldwide and used in this research.

Stony Brook University is a member of the SMARTS consortium, which operates the small telescopes at CTIO under contract to the NSF. The ANDICAM data were taken by service observers J. Espinosa and D. Gonzales.

C.B. was supported, in part, by the Deutsches Zentrum für Luft- und Raumfahrt (DLR), Förderkennzeichen 50 OW 0205.

TOBS acknowledges support from a Thuringian State Scholarship and from a Scholarship of the Evangelisches Studienwerk e.V. Villigst.

A.B. was supported by DFG grant NE 515 / 13-1 and 13-2.

\section{References}

Appenzeller, I., Mundt, R., \& Wolf, B. 1978, A\&A, 63, 289

Batalha, C., Lopes, D. F., \& Batalha, N. M. 2001, ApJ, 548, 377

Bertout, C., Wolf, B., Carrasco, L., \& Mundt, R. 1982, A\&AS, 47, 419

Boss, A. P. 2006, ApJ, 637, L137

Broeg, C., Fernández, M., \& Neuhäuser, R. 2005, Astron. Nachr., 326, 134

Burke, E. W., Rolland, W. W., \& Boy, W. R. 1970, JRASC, 64, 353

Chauvin, G., Lagrange, A.-M., Dumas, C., et al. 2005a, A\&A, 438, L25

Chauvin, G., Lagrange, A.-M., Zuckerman, B., et al. 2005b, A\&A, 438, L29

Close, L. M., Lenzen, R., Guirado, J. C., et al. 2005, Nature, 433, 286

Covino, E., Terranegra, L., Franchini, M., Chavarria-K., C., \& Stalio, R. 1992, A\&AS, 94, 273

Debes, J. H., \& Sigurdsson, S. 2006, A\&A, 451, 351

Dworetsky, M. M. 1983, MNRAS, 203, 917

Gregory, S. G., Jardine, M., Simpson, I., \& Donati, J.-F. 2006, MNRAS, 371, 999

Guenther, E. W. 2006, Rev. Mod. Astron., GQ Lup and its companion, ed. S. Röser (Berlin: Wiley-VCH), 19, 237

Guenther, E. W., Neuhäuser, R., Wuchterl, G., et al. 2005, Astron. Nachr., 326, 958

Guenther, E. W., Esposito, M., Mundt, R., et al. 2006, A\&A, submitted

Herbst, W. 1985, http://www.astro.wesleyan.edu/ bill/research/ ttauri.html

Herbst, W. 1989, AJ, 98, 2268

Hoffmeister, C. 1965, Veröffentlichungen der Sternwarte Sonneberg, 6, 97

Horne, J. H., \& Baliunas, S. L. 1986, ApJ, 302, 757

Jardine, M., Cameron, A. C., Donati, J.-F., Gregory, S. G., \& Wood, K. 2006, MNRAS, 367, 917

Johnson, H. L., \& Mitchell, R. I. 1975, Rev. Mex. Astron. Astrofis., 1, 299

Kardopolov, V. I., \& Filipev, G. K. 1985, Peremennye Zvezdy, 22, 103

Kenyon, S. J., \& Hartmann, L. 1995, ApJS, 101, 117

König, B., Guenther, E. W., Woitas, J., \& Hatzes, A. P. 2005, A\&A, 435, 215

Krautter, J., Wichmann, R., Schmitt, J. H. M. M., et al. 1997, A\&AS, 123, 329

Melo, C. H. F. 2003, A\&A, 410, 269

Mugrauer, M., \& Neuhäuser, R. 2005, Astron. Nachr., 326, 701

Mundt, R., \& Bastian, U. 1980, A\&AS, 39, 245

Murdoch, K. A., Hearnshaw, J. B., \& Clark, M. 1993, ApJ, 413, 349

Neuhäuser, R., Sterzik, M. F., Schmitt, J. H. M. M., Wichmann, R., \& Krautter, J. 1995, A\&A, 297, 391

Neuhäuser, R., Wolk, S. J., Torres, G., et al. 1998, A\&A, 334, 873

Neuhäuser, R., Guenther, E. W., Wuchterl, G., et al. 2005, A\&A, 435, L13

Paulson, D. B., Saar, S. H., Cochran, W. D., \& Hatzes, A. P. 2002, AJ, 124, 572

Paulson, D. B., Cochran, W. D., \& Hatzes, A. P. 2004, AJ, 127, 3579

Saar, S. H., \& Donahue, R. A. 1997, ApJ, 485, 319

Saar, S. H., Butler, R. P., \& Marcy, G. W. 1998, ApJ, 498, L153

Scargle, J. D. 1982, ApJ, 263, 835

Schwartz, R. D., \& Noah, P. 1978, AJ, 83, 785

Seifahrt, A., Neuhäuser, R., \& Hauschildt, P. H. 2007, A\&A, in press

Waagen, E. 2005, private communication 